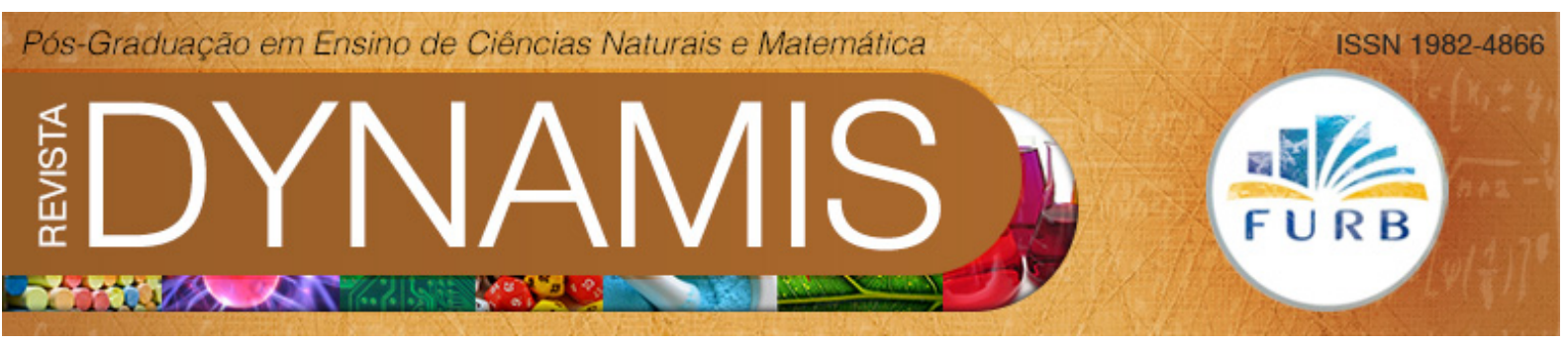

\title{
Potencial social de articulação entre ensino médio e a engenharia articulação universidade e ensino médio: as potencialidades das geotecnologias e 0 conhecimento científico na escola
}

Potential social articulation between high school and engineering college and high school articulation: the potential of geo-technologies and scientific knowledge in school

Tânia R. D. S. Pereira

Universidade Do Estado Da Bahia - UNEB tanreg@uneb.br

Fabiana S. Nascimento

Universidade Do Estado Da Bahia - UNEB

fabi30_nascimento@hotmail.com

Inaiá Brandão Pereira

Universidade Do Estado Da Bahia - UNEB inaiabrandao18@gmail.com

Telma D. S. dos Anjos

Universidade Do Estado Da Bahia - UNEB telmadias@uneb.br 
Potencial social de articulação entre ensino médio e a engenharia articulação universidade e ensino médio: as potencialidades das geotecnologias e o conhecimento científico na escola 


\title{
Resumo
}

Parece estar cada vez mais evidente a necessidade de promover ações de integração universidade-escola com o objetivo de derrubar as barreiras entre esses dois níveis de ensino e despertar vocações para as ciências exatas e a engenharia, contribuindo assim, para a melhoria da qualidade do ensino médio e promovendo a familiarização da sociedade com a tecnologia. Propomos nesse artigo descrever uma experiência entre a Universidade e o ensino médio, tendo como objetivo possibilitar aos alunos desse nível de ensino da rede pública, a exploração das potencialidades das Geotecnologias (dimensão técnica e subjetiva), enfatizando a pesquisa como eixo gerador à formação educacional e profissional desses sujeitos. A metodologia utilizada foi à abordagem qualitativa, tendo como estratégia metodológica a pesquisa de campo, onde foram incorporadas também visitações, cursos, palestras, encontros e oficinas. Como resultados, esperamos alargar as discussões e reflexões acerca do uso das TIC na formação educacional e científica dos alunos envolvidos, despertando-os para o ensino superior, bem como na escolha de sua formação profissional.

Palavras-chave: Integração, Geotecnologias, Tecnologias, Educação.

\begin{abstract}
Seems increasingly clear the need to promote actions to integrate university-school with the goal of breaking down barriers between these two levels of education and awakening vocations to the exact sciences and engineering, thus contributing to improving the quality of school and promoting society's familiarization with technology. We propose in this article describe an experiment between the University and the high school, aiming to enable students to this level of education of the public, exploiting the potential of Geotechnology (technical dimension and subjective), emphasizing research as the generator shaft and educational these professional subjects. The methodology was qualitative approach, with the methodological strategy research field, where they were also incorporated visits, courses, lectures, meetings and workshops. As a result, we expect to broaden the discussions and reflections on the use of ICT in the educational and scientific students involved, raising them to higher education as well as vocational training of their choice.
\end{abstract}

Keywords: Integration, Geotechnologies, Technology, Education. 


\section{Introdução}

$\mathrm{Na}$ sociedade contemporânea os temas ciência e tecnologia são extremamente discutidos nas diversas áreas do conhecimento, suplantando as barreiras existentes entre as mesmas e possibilitando uma rede de articulações. No entanto, mesmo com a popularização, essas temáticas são pouco desenvolvidas nas escolas, o que acaba acarretando na formação insuficiente de profissionais capacitados para atuarem na criação e produção de tecnologia.

Nesse contexto, é de basilar importância fomentar a pesquisa desde a Educação Básica, afinal, é através da pesquisa científica que é possível desenvolver autonomia e autoria, superando os modelos tradicionais de transmissão e reprodução de informações, que muitas vezes, buscam preparar indivíduos para a entrada em cursos superiores, mas não obtêm sucesso no que diz respeito ao preparo para a vida e os obstáculos impostos por ela. Em relação ao preparo dos estudantes para a entrada na universidade, podemos afirmar que o mesmo, apesar de válido, é feito, na maioria das vezes, para remediar atrasos e a falta de qualidade identificada durante a educação básica. A partir disso, é possível inferir que a educação formal escolar necessita ser potencializada, para que as práticas de ensino sejam redimensionadas.

No âmbito desse artigo, traremos a Educação Científica como um processo capaz de proporcionar melhorias no que tange a qualidade do ensino escolar, enfatizando no Ensino Médio como lócus para tal posição.

\section{Conhecimento científico-tecnológico no ensino médio}

Segundo a Lei de Diretrizes e Bases da Educação Nacional, o Ensino Médio "tem por finalidades desenvolver o educando, assegurar-lhe a formação comum indispensável para o exercício da cidadania e fornecer-lhe meios para progredir no trabalho e em estudos posteriores" (Art.22, Lei $n^{0}$ 9.394/96). Isso significa que o Ensino Médio é a etapa final da educação básica e deve ter por objetivo a preparação do indivíduo para a compreensão e o desenvolvimento pleno dos fundamentos científicos e tecnológicos presentes no processo produtivo da sociedade, além de competências para alcançar níveis mais complexos de ensino, passando por essa etapa de forma eficaz, consistente e crítica.

Os sujeitos imersos na Sociedade da Informação sentem cada vez mais a necessidade de interação com os outros e com o mundo, afinal, a própria dinâmica dessa sociedade da qual fazemos parte nos impulsiona a isso, pois é extremamente caracterizada pela conexão ilimitada e pela ultrapassagem dos limites do espaço e do tempo.

A complexidade do momento atual torna os fundamentos educacionais ainda mais enredados. O saber está cada vez mais fluído, visto que as informações estão sendo produzidas cada vez mais rápidas, bem como difundidas numa velocidade ainda maior. As TIC - Tecnologias da Informação e Comunicação transformaram os espectadores em atores e estes em co-autores de uma peça, onde cada ponto forma uma parte da teia do conhecimento. "Pela primeira vez na história da humanidade, a maioria das competências adquiridas por uma pessoa no início de seu percurso profissional estarão obsoletas no fim de sua carreira". (LEVY, 1999, p.157).

As palavras de Lévy (1999) deixam claro que o saber passa por renovações sucessivas e esse fenômeno deve ser discutido e tratado também pela escola. Complementando isso, colocamos que a interação entre o conhecimento sempre revigorado, o exercício da cidadania e a formação para realização de atividades produtivas reiteram o papel da educação como elemento de desenvolvimento social (PCN, p.11). 
Portanto, não podemos postergar a relevância do Ensino Médio na formação dos sujeitos na contemporaneidade, visto que o mesmo deve expressar essa era caracterizada pela contínua condição de mudança e rupturas.

E o que torna a educação um grande palco para mudanças na sociedade? Essa pergunta, se bem analisada, pode render uma tese, mas respondendo diretamente podemos afirmar que essa característica da educação é movida, essencialmente, pela sua progressiva possibilidade de reinvenção do conhecimento.

E é exatamente essa reinvenção do conhecimento que torna a Educação Científica um processo tão relevante na atualidade, pois possibilita não apenas a apreensão de informações e a transformação delas em conhecimento, mas a possibilidade de utilizar esse saber para prospectar oportunidades dinâmicas e produtivas.

A Educação Científica possibilita o que poderíamos chamar de desenvolvimento da inteligência, pois a inteligência é algo intrínseco ao ser humano, mas a utilização consciente dessa habilidade é algo que, na maioria das vezes, é formado pela sociedade. O processo supracitado tem como preceitos a pesquisa, o estudo aprofundado e a produção de conhecimento:

O aluno não está condenado a copiar coisa copiada. Pode também, dentro de suas limitações naturais, exercitar textos científicos, com o objetivo de tornar-se capaz de produção própria, o que lhe permite continuar aprendendo e se atualizando a vida toda. Uma coisa é absorver conteúdos, outra, bem diferente, é reconstruí-los, investindo neste processo alguma originalidade. (DEMO, 2010, p. 16).

Autoria e autonomia são palavras decisivas nos processos formativos dos indivíduos educados cientificamente, visto que a elaboração de conhecimento inovador é o ponto "chave" para o desenvolvimento de uma sociedade e a pesquisa é, a nosso ver, a melhor forma de alcançá-lo.

Salientamos que a tríade educação-pesquisa-inovação pode acarretar diversos benefícios no que tange o desenvolvimento científico e tecnológico do país. Nessa perspectiva, é de salutar relevância considerar o Ensino Médio um período decisivo na vida do estudante, pois é o momento onde estão concentradas as expectativas para outros níveis de ensino, sejam eles superior ou técnico.

\section{Geotecnologias: conceitos e aplicações}

A educação na contemporaneidade exige novas formas de pensar, analisar, concluir, inferir e interpretar, provocando constantes transformações no processo de ensino e aprendizagem. Segundo Freire e Valente (2001), o uso da tecnologia com finalidade pedagógica visa principalmente à integração dos alunos e professores, buscando compreender e interpretar fenômenos socioculturais bem como o envolvimento em atividades sociais relevantes. No ensino e aprendizagem da Engenharia, o advento da Geotecnologia delimita novos caminhos e remodela as práticas de ensino e a compreensão dos elementos que constituem o espaço.

A literatura aponta que a geotecnologia é a utilização da informação para a análise do espaço geográfico, realizada por meio da tecnologia. São compreendidas como os processos humanos e técnicos que os sujeitos utilizam para conhecer, representar e estudar os espaços da terra (NASCIMENTO, 2010, p.18). Ou seja, são as geotecnologias que nos permitem utilizar diferentes técnicas como os satélites, as fotografias aéreas e outras tecnologias digitais capazes de indicar novos caminhos para desenvolver novas práticas de ensino sobre a compreensão dos elementos que constituem o espaço vivido dos sujeitos alunos, meninos, homens e mulheres, entre demais espécies. 
Embora com caráter técnico, as geotecnologias apresentam aspectos social, antropológico, emocional, político e econômico (LOBÃO E CHAVES, 2008, p. 35), denotando assim que a utilização das suas potencialidades nos processos formativos ensinoaprendizagem representa uma nova perspectiva e uma nova possibilidade de inclusão, pois além de poder inserir o sujeito no mundo digital permite a construção de uma compreensão acerca do espaço geográfico, fortalecendo os enlances de pertencimento, de conhecimento e de cidadania. (BRITO E HETKOWSKI, 2010, p. 61). Ainda de acordo com Hetkowski: “... tecnologias são processos humanos criativos, que envolvem elementos materiais (instrumentos e técnicas) e imateriais (simbólicos e cognitivos) e que se encarnam na linguagem do saber e do fazer dos homens"(2010, p.6).

Nos cursos de engenharia essas habilidades devem ser ressaltadas, pois segundo as Diretrizes Curriculares dos Cursos de Engenharia no Artigo $3^{\circ}$ :

O Curso de Graduação em Engenharia tem como perfil do formando egresso/profissional o engenheiro, com formação generalista, humanista, crítica e reflexiva, capacitado a absorver e desenvolver novas tecnologias, estimulando a sua atuação crítica e criativa na identificação e resolução de problemas, considerando seus aspectos políticos, econômicos, sociais, ambientais e culturais, com visão ética e humanística, em atendimento às demandas da sociedade.(CNE, 2002).

Nesse contexto emergem as geotecnologias como uma alternativa de recurso didático a ser utilizado no ensino e aprendizagem, haja vista a atratividade, principalmente pelo fato de proporcionar grande interatividade entre o estudante e o objeto de estudo, mediada pelo docente. Novas possibilidades na utilização das geotecnologias são reveladas, que não aquelas voltadas à produção, ao controle e a circulação, mas relacionadas à compreensão social do espaço, voltadas também a inclusão do espaço no sentido de um processo social de construção e apropriação do espaço (HETKOWSKI, 2011, p. 10). Desta forma, as geotecnologias permitem o entrelaçamento entre as técnicas e a criatividade humana, através da exploração e utilização de imagens de satélite, fotografias aéreas, Google Earth e Maps, ferramentas, etc. As geotecnologias também são utilizadas no apoio aos diversos serviços nas diversas modalidades de engenharia, a exemplo da construção civil (topografia, localização, cadastros, fundações, mapeamentos, etc.).

\section{Núcleo de geotecnologias, educação e contemporaneidade - Geotec}

A sociedade demanda de forma cada vez mais evidente que a Universidade deve promover ações de integração universidade-escola de ensino médio com o objetivo de despertar vocações para as ciências exatas e a engenharia. Contribuindo assim, para a melhoria da qualidade do ensino médio e promovendo a familiarização da sociedade com a tecnologia. Com vistas à essa necessidade, destacamos neste trabalho, às atividades realizadas junto aos estudantes do Ensino Médio do Colégio da Polícia Militar do Estado da Bahia - CPM, cujo objetivo principal é o de promover o conhecimento científico no ambiente escolar e incentivar o interesse dos jovens quanto à apropriação dos conhecimentos tecnológicos. Esta intenção parte da reconhecida importância da formação técnica para a atuação profissional em nossa sociedade, tendo em vista a demanda por pessoas qualificadas nos diversos setores do mercado de trabalho.

Com essa visão e a partir da perspectiva de elaborar estudos acerca das geotecnologias e suas potencialidades no ensino, iniciamos no ano de 2007 as atividades do grupo de pesquisa GEOTEC, coordenado pela Dra. Tânia Maria Hetkoswki na Universidade do Estado da Bahia - UNEB. Nestes quatro anos o núcleo desenvolveu pesquisas que abrangem desde a formação de professores, no que tange ao uso de geotecnologias em sua prática pedagógica, à iniciação científica e tecnológica junto aos alunos de ensino médio da rede pública de ensino de Salvador. Desta forma, iniciamos em 2010 o projeto intitulado de "Geotecnologias: 
conhecendo o lugar e entendo o mundo", em parceria com o CPM-BA. O objetivo deste projeto foi de proporcionar aos estudantes habilidades, através dos recursos das geotecnologias para analisar aspectos referentes a dos fenômenos urbanos a partir da análise de alguns bairros de cidade do Salvador-BA, abordando sua história, problemas e diversas características.

Desenvolvemos os estudos através dos seguintes procedimentos: seleção de quatro bairros da cidade de Salvador-BA, situados em pontos distintos da cidade; coleta de informações sobre os mesmos através do levantamento de materiais cartográficos (mapas, fotografias aéreas e imagens orbitais - recentes e antigas) e realização de entrevistas, busca de reportagens e textos acadêmicos; além da exploração dos recursos das geotecnologias disponíveis no programa Google Earth e no Google Maps, a fim de subsidiar a análise dos bairros.

Sendo assim, elaborou-se uma análise dos bairros de Boca do Rio, Cabula, Periperi e Vila Laura, identificando as principais transformações sócio-espaciais das referidas localidades de Salvador, bem como diversos problemas, dentre os quais podemos destacar: a poluição de rios e mananciais; desmatamento, especulação imobiliária, uso e ocupação do solo. Assim, os estudantes identificaram a construção de novos prédios e as áreas que ocorreram maiores mudanças nos bairros, resultado do processo de especulação e crescimento do setor imobiliário, fato evidenciado também com a pesquisa de campo efetuada na localidade. Análises e estudos como estes possibilitaram uma aproximação, mesmo que de forma simples, dos estudantes com as possibilidades propiciadas pelas geotecnologias para a realização de estudos sobre a cidade, tanto para construção de diagnósticos, como para fins de planejamento.

Outro objetivo do projeto foi o de proporcionar aos estudantes o conhecimento acerca da atuação de profissionais que utilizam como ferramentas de trabalho a cartografia e as geotecnologias, a exemplo dos engenheiros, arquitetos, urbanistas e geógrafos. Com esta perspectiva os pesquisadores visitaram o setor de cartografia da Companhia de Desenvolvimento do Estado da Bahia - CONDER, no qual puderam conhecer como são feitos os mapeamentos do Estado da Bahia e suas aplicabilidades para fins de planejamento urbano.

Assim estabelecendo uma aproximação dos jovens pesquisadores com essas áreas profissionais, espera-se despertar o interesse pela atuação profissional nas atividades relacionadas às mesmas. Vale-se ressaltar que o atual contexto do Brasil, sediando eventos importantes como a Copa do Mundo e as Olimpíadas, deu notoriedade as áreas de atuação ligadas à construção civil e planejamento urbano, demonstrando a importância destas para o desenvolvimento do país. Tal fato deve-se principalmente à adequação das cidades (em relação a setores como transporte público) para atender as demandas de tais eventos, o que abriu e ampliou às discussões a cerca das carências de infra-estrutura das cidades brasileiras. Tais discussões vêm provocando o interesse dos jovens e estudantes do Ensino Médio e de Graduação, tornando-se uma ótima oportunidade de instigá-los em um processo de formação técnico-científica.

Neste sentido, vemos de grande urgência a necessidade de discussão que traga a tona que, ciência e tecnologia, são atividades humanas de grande importância social, pois são responsáveis pela formação de parte da cultura geral nas sociedades modernas (BAZZO, 2011).

Com isso a universidade corresponde a sua responsabilidade, disponibilizando aparatos tecnológicos, com o objetivo de demonstrar para os alunos a importância que tais profissões têm para a sociedade e desenvolvimento do país, contribuindo para formação de uma 34 
consciência crítica, de cidadania, ofertando assim "o desenvolvimento de capacidades cognitivas especialmente orientadas para uma nova compreensão da natureza do fenômeno científico tecnológico e de seus produtos, considerando também as diferenças intersociais" (Von LINSINGEN, 2006, p.6).

\section{Considerações finais}

Além da formação e capacitação dos estudantes para o desenvolvimento pessoal e profissional que foram viabilizados através dos encontros temáticos, que objetivaram fornecer aos participantes a aquisição de novos conhecimentos e desenvolvimento de habilidades por meio do contato com novas abordagens teóricas, exercícios e experiências vivenciadas.

A articulação entre Universidade e Ensino Médio, seja na formação docente quanto na formação profissional e cidadã do aluno, contribui para redução das deficiências que os ingressantes da educação superior apresentam, bem como, desperta para as vocações científicas e tecnológicas.

Consideramos que essa experiência ofereceu aos estudantes do ensino médio, subsídios técnicos, tecnológicos, teóricos e metodológicos, proporcionando-os, assim, auxiliar e despertar a vocação profissional dos mesmos nas diversas áreas do conhecimento e principalmente para as engenharias.

\section{Referências}

BAZZO, W.A. Ciência, Tecnologia e Sociedade e o contexto da educação tecnológica. 3 ed. Florianópolis: EdUFSC, 2011.

BRITO, F. J. de O.; HETKOWSKI, T. M. Geotecnologias: possibilidades de inclusão sócioespacial. In: BONETI, L. W.; ALMEIDA, N. P.; HETKOWSKI, T. M. (Org.). Inclusão digital: da teoria à prática. Curitiba: Imprensa Oficial, 2010.

DEMO, Pedro. Educação científica. B. Téc. SENAC: a R. Educ. Prof., Rio de Janeiro, v. 36, n.1, jan./abr. 2010. Disponível em: www.senac.br/BTS/361/artigo2.pdf. Acesso em: 06 jul 2011.

FREIRE, F. M. P.; VALENTE, J. A. Aprendendo para a vida: os computadores na sala de aula. São Paulo: Cortez, 2001.

HETKOWSKI, T. M. Geotecnologias: como explorar educação cartográfica com novas gerações? In: XV Encontro Nacional de Didática e Prática de Ensino (ENDIPE), 2010, Belo Horizonte. Anais... Belo Horizonte, MG: UFMG, 2010.

LÉVY, P. Cibercultura. São Paulo: Editora 34, 1999.

LOBÃO, J. S. B; CHAVES, J. M. Geotecnologias na aprendizagem da geografia: alternativas para inclusão digital. Geografia's. Feira de Santana, n. 1, p. 35-40, mai/nov, 2008.

MEC. Resolução CNE/CES nº 11, de 11 de março de 2002. Diretrizes Curriculares Nacionais do Curso de Graduação em Engenharia. MEC, 2002.

Secretaria de Educação Básica. Parâmetros Curriculares Nacionais: Ensino Médio. Brasília: MEC / SEF, 2000.

Conselho Nacional de Educação. Diretrizes Curriculares Nacionais para o Ensino Médio. Brasília: MEC, 1998. 
NASCIMENTO, F. dos S. Potencialidades da Educação Cartográfica nos anos iniciais do ensino fundamental: a importância da formação de professores. Monografia (graduação). Departamento de Educação, Universidade do Estado da Bahia, Salvador, 2010. 59f.

Von LINSINGEN, I. CTS na educação tecnológica: tensões e desafios. Congresso Iberoamericano de Ciencia, Tecnologia, Sociedad e Innovacción - CTS+I. Palácio de Minería, 2006. p. 1-13. 